www.jmscr.igmpublication.org

Impact Factor 5.84

Index Copernicus Value: 71.58

ISSN (e)-2347-176x ISSN (p) 2455-0450

crossref DOI: _https://dx.doi.org/10.18535/jmscr/v6i1.120

\author{
Journal Of Medical Science And Clinical Research \\ IGM Publication \\ An Official Publication of IGM Publication
}

\title{
Chronic Osteomyelitis in Adults: A Prospective Study
}

\author{
Authors \\ Dr Vishwas Sharad Phadke ${ }^{1}$, Dr Satish Divakar Mehta ${ }^{2}$, Dr Sunil V.Patil ${ }^{3}$ \\ ${ }^{1,2}$ Assistant Professor in Orthopaedics, Bharati Vidyapeeth Deemed University Medical College, Sangli \\ (Maharashtra). - India \\ ${ }^{3}$ Prof. \&HOD, Dept of Orthopaedics, Bharati Vidyapeeth Deemed University Medical College, Sangli \\ (Maharashtra) - India \\ Corresponding Author \\ Dr Satish Divakar Mehta \\ Assistant Professor in Orthopaedics, Bharati Vidyapeeth Deemed University Medical College, Sangli \\ (Maharashtra) India
}

\begin{abstract}
Background: Osteomyelitis is inflammation of the bone caused by an infecting organism. It is divided into acute, subacute and chronic osteomyelitis on the basis of the duration from the onset of symptoms to the definitive diagnosis. The common predisposing factors for development of chronic osteomyelitis are diabetes, immunosuppression, individuals on immunosuppressants, chemotherapeutic agents or on steroids. The management of chronic osteomyelitis is challenging and consist of prolonged course of appropriate antibiotics and surgical interventions (skin or bone grafting, debridement, drainage of pus and amputation in unrelenting infections).

Aims and Objectives: (1) To study the factors predisposing an individual for the development of chronic osteomyelitis.

(2) To know the common bones and type of organisms involved in chronic osteomyelitis.

(3) To study the outcome of cases after appropriate management.

Methods: This was a prospective study in which patients of more than 18 years of age and having been diagnosed with chronic osteomyelitis were enrolled on the basis of predefined inclusion and exclusion criteria. The institutional ethical committee approved the study. Informed consent was taken from all the patients. Demographic details, clinical features, associated comorbidities and involved bone were all noted down in a predefined proforma. Diagnosis was confirmed by imaging and culture sensitivity. Patients were managed by surgical interventions and prolonged antibiotics. Patients were followed up for 1 year and outcome was studied.

Results: Out of 45 studied cases males were affected predominantly with a M: F ratio of 1:0.6. Most common age group affected was found to be between $41-50$ years. Most common bones involved were femur (28.88\%) and tibia (26.66\%) followed by fibula (11.11\%), iliac bones (6.66\%) and humerus (6.66 \%). In most of the cases (35/45) contagious spread following trauma was the mechanism of infection. Comorbidities like hypertension, diabetes and immunosuppression was present in $44.44 \%$ patients. S. Aureaus (17/45) followed by Pseudomonas (9/45) or enterococci (6/45) were the most common pathogens isolated by appropriate culture methods. Finally the analysis of outcome showed that 28 patients were completely cured while remaining patient had some or the other problem associated with chronic osteomyelitis. 3 patients died during study period due to causes unrelated to osteomyelitis.

Conclusion: Management of chronic osteomyelitis is a challenge for treating orthopedicians. Appropriate surgical interventions and suitable antibiotics for prolonged periods are required. Inadequate treatment is fraught with the danger of complications.

Keywords: Chronic Osteomyelitis, risk factors, Management, Outcome.
\end{abstract}




\section{Introduction}

Osteomyelitis is defined as inflammation of the bone caused by an infecting organism ${ }^{1}$. In healthy individuals bone tissue is usually resistant to infecting organisms but in certain conditions like trauma, foreign body, surgery, implants and immunosuppression infection may get hold of bone tissue. Once established the further course and severity of infection depends upon virulence of infecting organisms, immune status of the affected individual and location of the affected bone $^{2}$. There are 2 basic mechanisms by which infections reach the bone namely contagious spread and haematogenus spread. Incases of contagious spread the infection usually reaches to the bone from direct inoculation of infecting organisms through trauma, surgical procedures, prostheses, spread from adjacent site of infection or nosocomial contamination. In cases of haematogenus spread the infection reaches the bone via haematogenus route ${ }^{3}$. This route of infection though more common in infants and children may also be seen in adults. The most common affected bones may include long bones, vertebrae, pelvis and clavicle ${ }^{4}$. Haematogenus spread of infection to bones causing osteomyelitis has been divided into primary and secondary haematogenus spread depending upon whether the remote infection was either a primary one or was result of reactivation of infection lying dormant since many years ${ }^{5}$. The common organisms found to be causing osteomyelitis include Staphylococcus, pseudomonas and enterobacteriaceae and streptococcus $^{6}$. Osteomyelitis is further divided into acute, subacute or chronic osteomyelitis on the basis of time between the onset of symptoms and the diagnosis. Acute osteomyelitis is defines as an infection where the period between the onset of symptoms and the diagnosis is less than 2 weeks. Subacute osteomyelitis is the one in which the time period between onset of symptoms and the diagnosis is between 2 weeks- 6 weeks and chronic osteomyelitis is the type of infection in which the diagnosis is generally delayed beyond 6-12 weeks ${ }^{7}$. The common risk factors for the occurrence of chronic osteomyelitis include trauma, operative procedures, malnutrition, chronic renal or hepatic failure, immunosuppression due to any cause, diabetes mellitus, vasculitis and radiation ${ }^{8}$. The diagnosis is usually suspected on the basis of history and clinical examination. Confirmation can be done by imaging (X-ray, CT or MRI) and culture sensitivity of the drained pus ${ }^{9}$. The management of chronic osteomyelitis is difficult and may depend on the factors like age and immune status of the patient, site and severity of the infection and type of organisms involved ${ }^{10}$. The principals of the management includes halting the progress of infection and eradicating it by use of appropriate antibiotics, reduce the symptoms (Pain and restricted movements) and retain the functionality of the affected $\operatorname{site}^{11}$. In resistant cases surgeries like sequestrectomy, debridement, soft tissue reconstruction and bone grafting may be needed. The fact that the individuals predisposed to chronic osteomyelitis require management of comorbid conditions like diabetes mellitus, immunosuppression and chronic organ failure make it necessary that a multidisciplinary treatment is instituted whenever necessary. Failure to properly treat this condition is fraught with danger of disseminated infection, sepsis and multi organ dysfunction ${ }^{12}$.

It is important to mention that at present no uniform clinical definition of chronic osteomyelitis and many clinicians and orthopedicians are using different criteria to define it making it difficult to compare and study different approaches in the management of chronic osteomyelitis. We conducted this study to know the risk factors, clinical features and management of chronic osteomyelitis.

\section{Materials and Methods}

This was a prospective study of patients presenting with chronic osteomyelitis. The study was approved by institutional ethical committee. All the patients of age more than 18 years who presented to department of orthopedics of a 
medical college located in an urban area with the signs and symptoms of osteomyelitis since more than 6 weeks and in whom the diagnosis was confirmed on the basis of imaging and culture sensitivity were included in this study. The demographic data like gender, age and predisposing factors were noted. Etiology and site of infection, signs and symptoms, causative organisms and outcome of treatment were studied. A detailed history was taken in all the patients specifically to find out the presence of predisposing factors. A thorough clinical examination including general, systemic and local examination was done. Imaging studies (X-ray, CT scan and MRI in selected patients) were done. The radiographic changes seen were studied. The involved bone, time since first symptom and diagnosis, mechanism of bone infection (Trauma, operation or presence of distant infective focus), bone specimen for culture, growth of organisms and their sensitivity patterns were all noted down. Antibiotics were preferably given on the basis of culture sensitivity reports and antibiotics were continued for 4-6 weeks. Outcome of the treatment was assessed during follow up visits to orthopedics OPD for at least 1 year.

In the statistical analysis of the patients in our study continuous variables were shown as mean for the parametric data and median if the data was nonparametric or skewed. Statistical analysis was done using Minitab version 17 running on windows 8. Microsoft word is used for manuscript preparation while excel was used for creation of figures and graphs.

\section{Inclusion Criteria}

- All the patients who have been diagnosed with osteomyelitis on the basis of appropriate imaging and culture results.

- The duration from onset of symptoms and the diagnosis was more than 6 weeks.

- Those who have given informed consent to be part of the study.

- Age more than 18 years

\section{Exclusion Criteria}

1. Age less than 18 years.

2. Those who refused informed consent.

3. The patients who didn't remain in follow up at least for 1 year.

\section{Results}

In this Prospective study of 45 patients with chronic osteomyelitis there were $28(62.22 \%)$ males and $17(37.77 \%)$ females with a M: F ratio of 1:0.6.

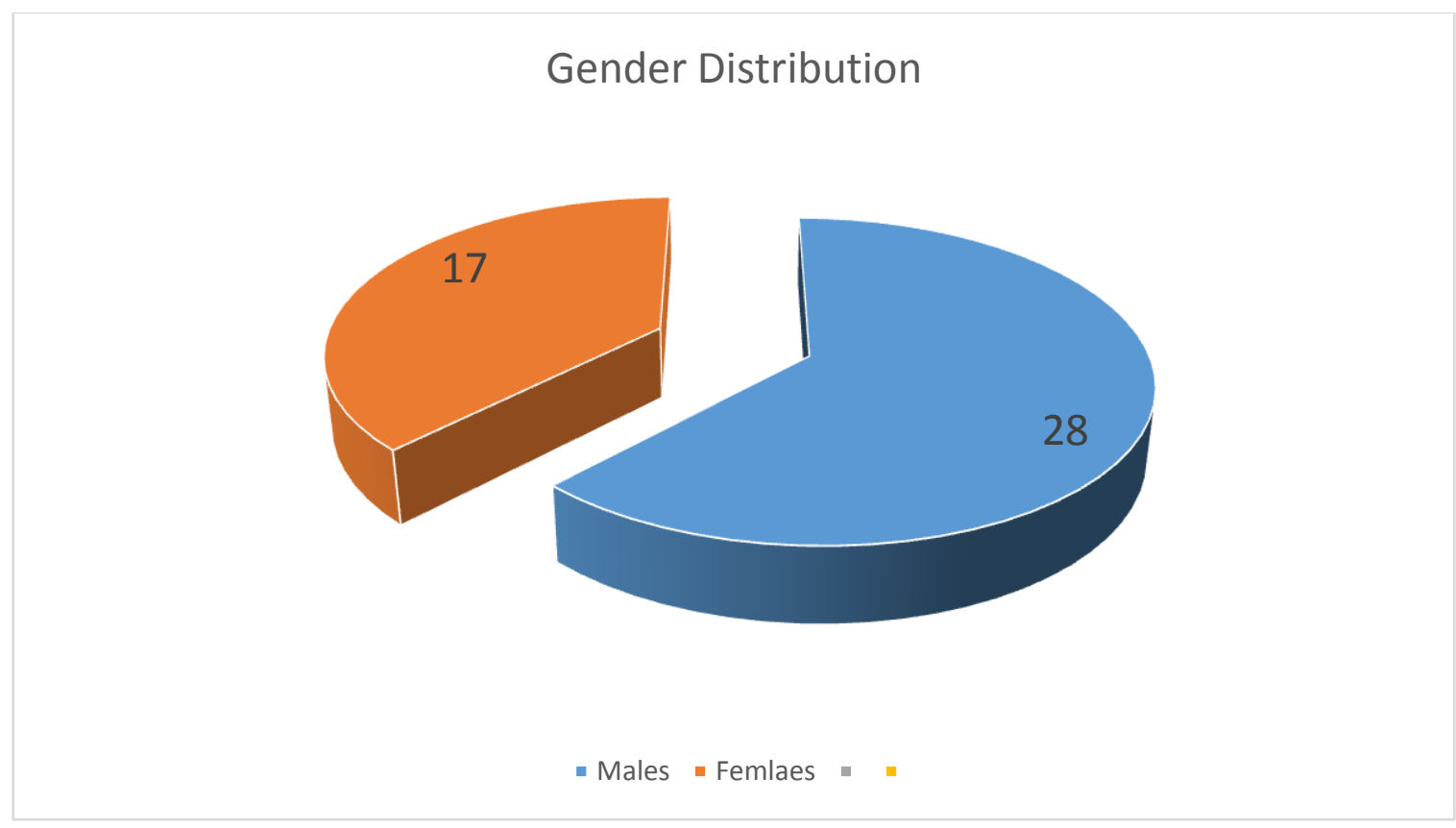

Figure 1: Gender Distribution of the studied cases. 


\section{JMSCR Vol||06||Issue||01||Page 32148-32156||January}

Study of the age groups of the patients showed that the most common age group affected by chronic osteomyelitis was found to be between the age group of 41-50 years followed by 50-60 years and 30-40 years.

Table 1: Age Group of the patients having chronic osteomyelitis

\begin{tabular}{|l|c|c|}
\hline Age Group & No Of Patients & Percentage \\
\hline $\mathbf{1 8 - 3 0}$ years & 6 & $13.33 \%$ \\
\hline $\mathbf{3 1 - 4 0}$ years & 7 & $15.55 \%$ \\
\hline $\mathbf{4 1 - 5 0}$ years & 18 & $40 \%$ \\
\hline $\mathbf{5 1 - 6 0}$ years & 14 & $31.11 \%$ \\
\hline
\end{tabular}

The analysis of the affected bone revealed that the most commonly affected bone was femur $(28.88 \%)$ followed by tibia $(26.66 \%)$, fibula $(11.11 \%)$, iliac bones $(6.66 \%)$, humerus $(6.66 \%)$ vertebrae $(4.44 \%)$ and other medullary bones $(15.55 \%)$.

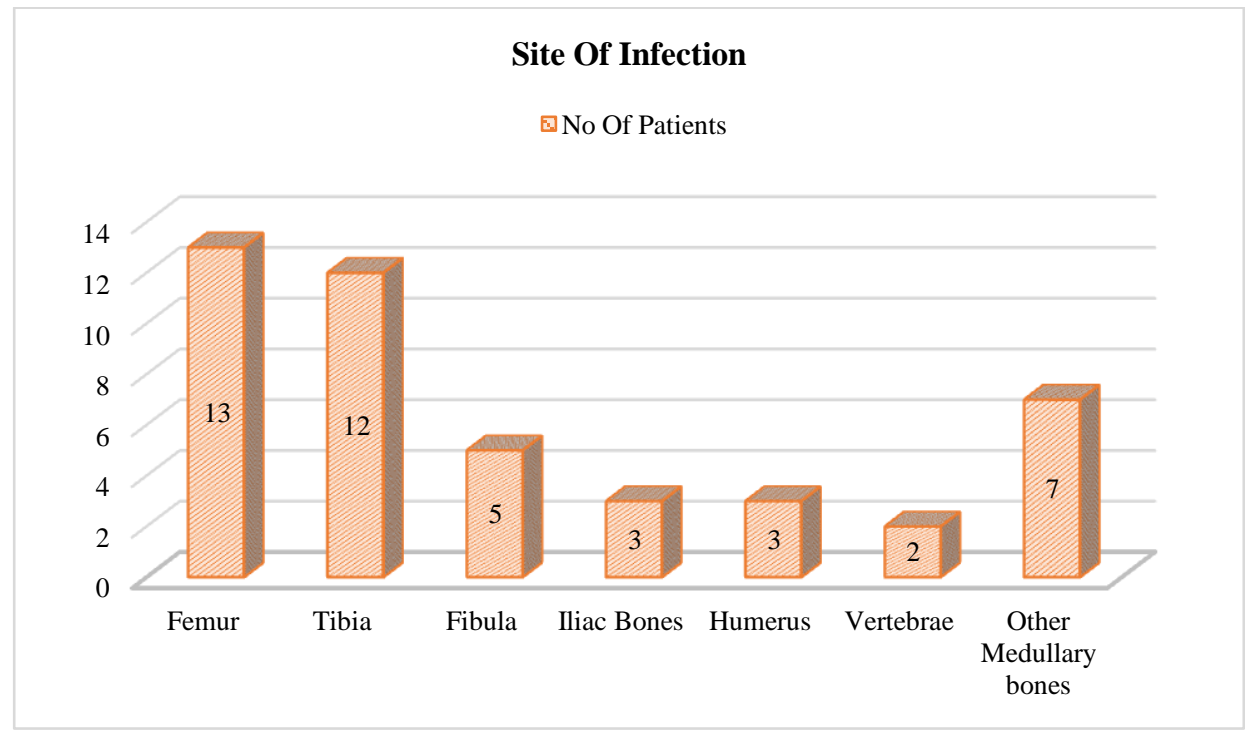

Figure 2: Site of infection in the studied cases.

In most of the cases (35/45) the infection reached the site of infection by local spread of infection from a contagious site. Out of the patients in whom the spread of infection was seen from a local site most of the patients acquired infection following trauma (29/35) while other causes of local spread included surgical procedures $(3 / 35)$ and spread of infection from skin (3/35). In remaining 10 patients chronic osteomyelitis was seen following haematogenus spread from a distant focus.

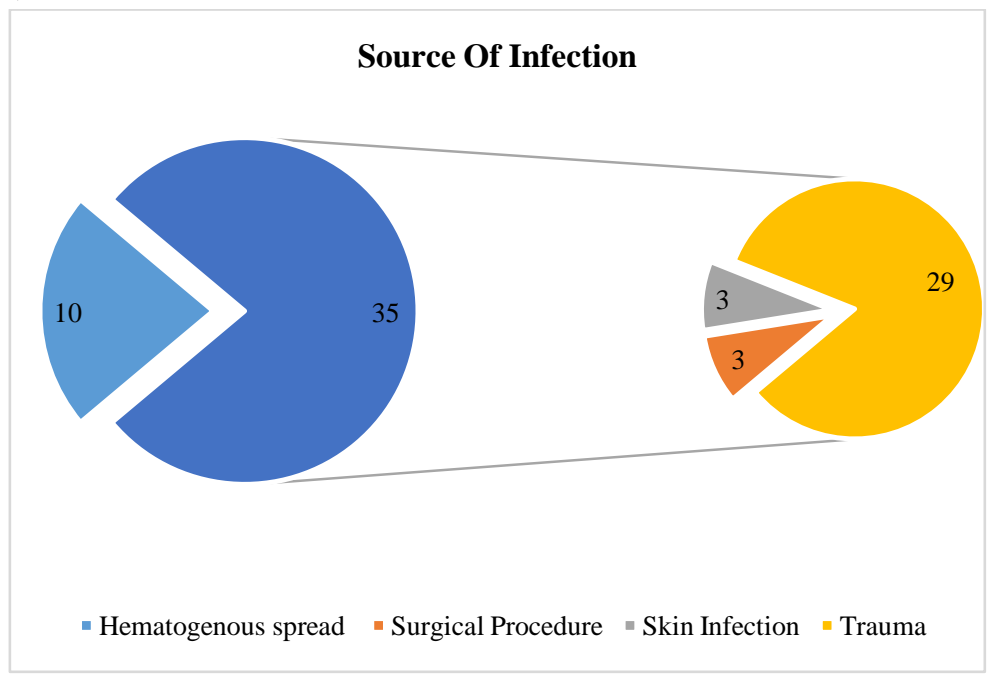

Figure 3: Mechanism of Infection 
Appropriate tissue was sent for culture and sensitivity in all the patients. Specimens such as bone biopsy (36), pus (2), affected soft tissue (3) and wound swabs (4) were sent for culture sensitivity.

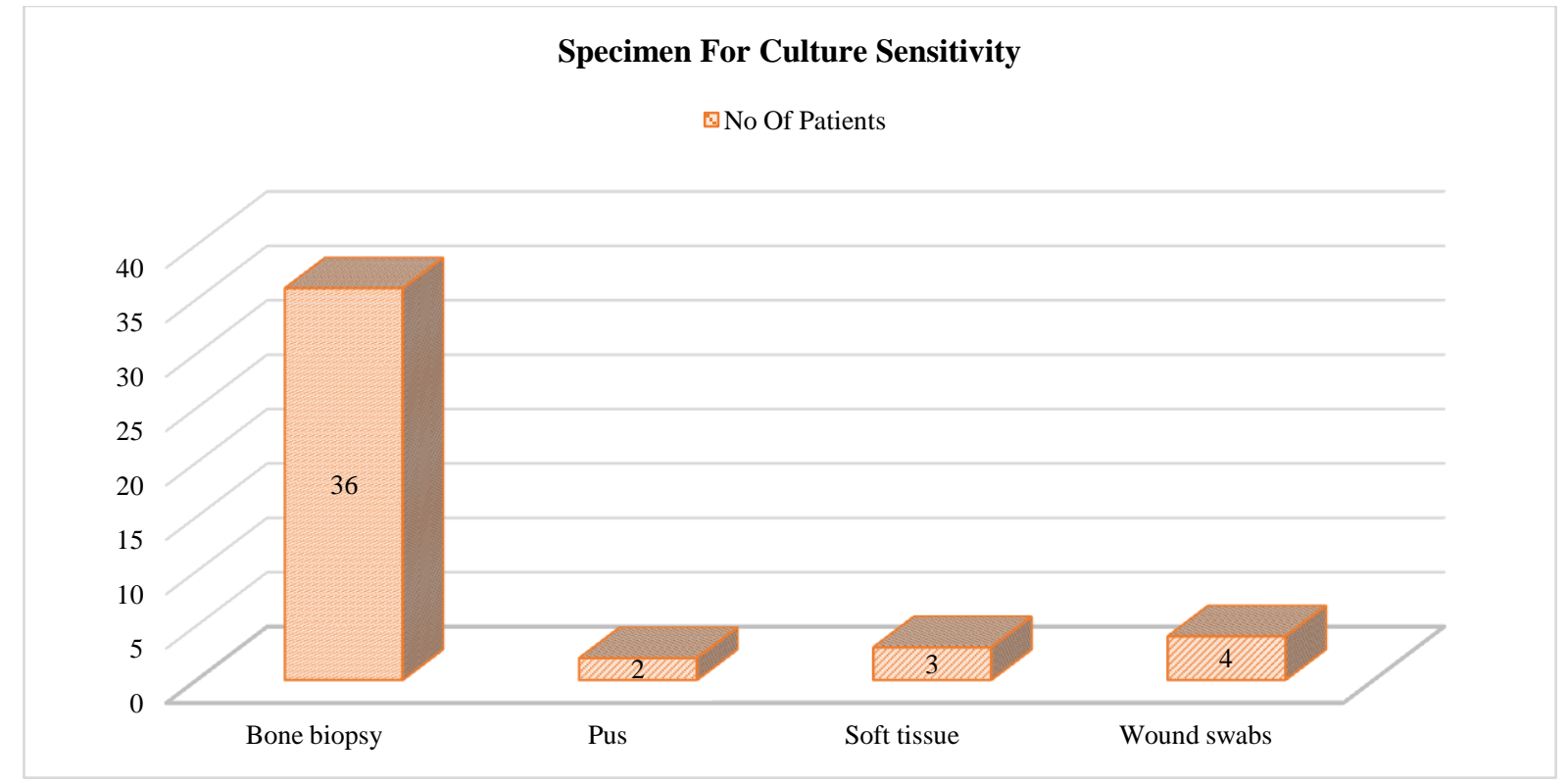

Figure 4: Culture specimen for culture and sensitivity tests.

The study of associated co-morbid conditions revealed that out of 45 patients 9 were having hypertension, 6 were diabetics, 3 patients were on immunosuprresants, 1 was on long term steroid therapy for nephrotic syndrome and 1 patient was having acquired immunodeficiency syndrome (AIDS).

\section{Co-Morbidities}

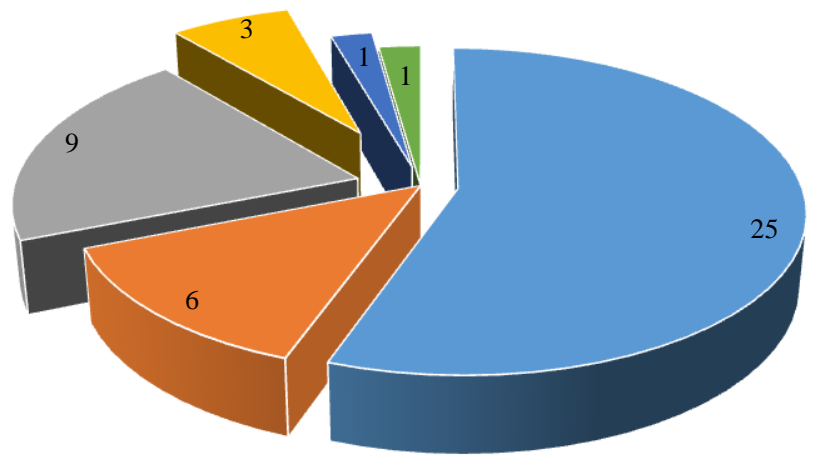

" No comorbidities = Diabetes " Hypertension =On immunosupressants = On steroid " AIDS

Figure 5: Presence of Associated comorbidities in studied cases

The analysis of organisms found in culture sensitivity of the studied cases showed that the most common offending organism was Staphylococcus Aureaus (17/45) followed by
Pseudomonas (9/45), enterococcus (6/45), Anaerobic bacterias (5/45), streptococcus (2/45) and other enterobactereciacea. 


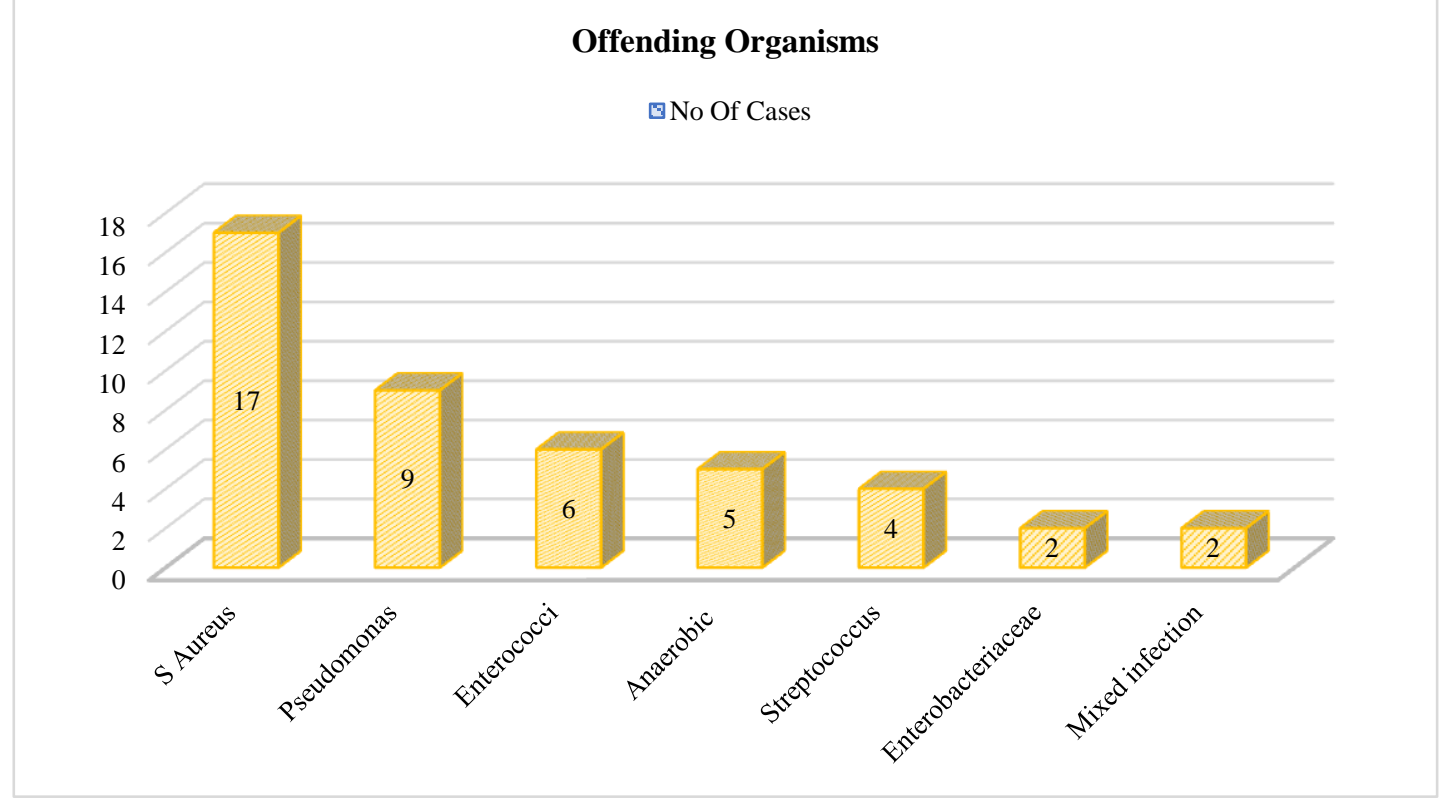

Figure 6: Offending organisms isolated in culture and sensitivity.

Management of the patient was done on the basis of site involved, severity of infection and associated co-morbid conditions. All patients received antibiotics at least for 4 weeks in addition to appropriate surgical management like debridement, pus drainage, skin and bone grafting. All patients responded well to the treatment 28 patients were completely cured and were not having any complaints during follow up period 5 had chronic pain 6 patients still had manifestation of chronic osteomyelitis in the form of pus discharge, recurrent fever or continued pain and 3 patient had to undergo some form of amputation due to chronic osteomyelitis and lastly 3 patients were expired during follow up due to reasons not related to osteomyelitis.

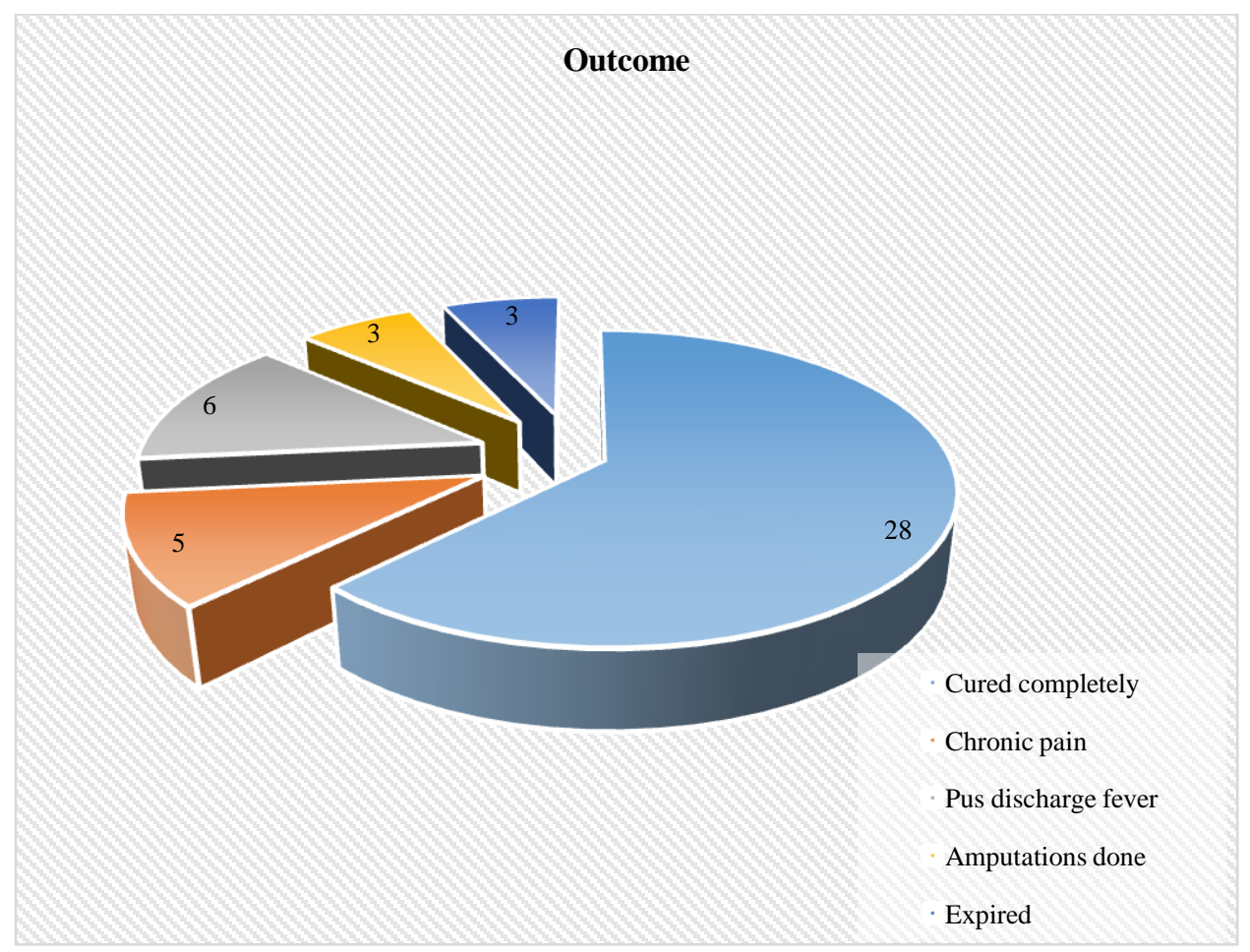

Figure 7: Outcome of patients with chronic osteomyelitis. 


\section{Discussion}

Management of chronic osteomyelitis is challenging even for most experienced orthopedicians. Bone is relatively avascular part of human body which makes it relatively resistant to infections but once the infection occurs then this very relatively avascular nature of bone becomes a hindrance in successful management of osteomyelitis $^{13}$. Certain predisposing factors like HIV, diabetes, individuals on long term steroids and immunosuppressive drugs and chemotherapeutic agents not only predisposes individuals for developing chronic osteomyelitis but also makes the treatment difficult and there are more chances of recurrences in these individuals in comparison to patients with competent immune system and with no comorbidities ${ }^{14}$.

In our study males were predominantly affected and male to female ratio was found to be 1:0.6. The fact that the most common cause of chronic osteomyelitis was found to be contagious spread following trauma makes it more likely that men will be affected more than the females because of involvement of men in activities like sports and road traffic accidents. Various studies have found men to be affected more than the females in cases of osteomyelitis. Lu Y Et al in their study of surgical management of chronic osteomyelitis found that $\mathrm{M}$ : F ratio in the studied cases was 3:2. Similarly another study conducted by Shen Y et al also showed that the males were predominantly affected in cases of traumatic osteomyelitis with a M:F ratio of $8: 1^{15}$.

The most common age group to have been affected by osteomyelitis was found to be between 41-50 years. Though in many instances the chronic osteomyelitis is a sequel to acute osteomyelitis especially in individuals with conditions such as diabetes or immunosuppression the fact that the single most reason for chronic osteomyelitis is trauma it is expected to be seen in individuals having an active life style making them prone for trauma. Various other studies have found similar results for example in a study of 24 patients with chronic osteomyelitis conducted by $\mathrm{Lu} \mathrm{Y}$ et al the average age was found to be 45.6 years. Various other studies reported slight variations in the most common affected age group but the common finding was that the active individuals were most likely to develop chronic osteomyelitis because of the increases intensity and severity of trauma ${ }^{16}$.

Femur and tibia were the most common bones to have been affected followed by fibula, iliacbones, humerus and vertebrae. Affection of tibia and fibula is not only common after the contagious spread from a local trauma site but also these bone are affected following haematogenus spread from a distant focus. Osteomyelitis resulting from haematogenus spread usually involves metaphysis of femur and tibia. Many authors have reported femur and tibia to be the common bones affected by osteomyelitis. Joseph M Fritz et al reported femur and tibia to be the most commonly affected bones in case of osteomyelitis secondary to haematogenus spread ${ }^{17}$.

The most common organisms cultured were S.Aureus followed by pseudomonas and enterococci. Various authors have uniformly reported S.Aureus to be the most common offending agent involved in acute as well as chronic osteomyelitis. Thanni L O et al in their review found staphylococcus to be the most commonly involved organism in patients of acute as well as chronic osteomyelitis in Africa and Middle east ${ }^{18}$. Similar findings were seen in the review done by IA Ikpeme et $\mathrm{al}^{19}$.

The management of chronic osteomyelitis is challenging and depends upon various factors which include age of the patient, site and severity of infection, immune status of the patient, extent of bone destruction and organisms involved. Usually prolonged antibiotic treatment (4-6 weeks), appropriate surgical intervention like surgical debridement, pus drainage, skin or bone grafting and in severe unrelenting cases amputations may be required ${ }^{20}$. 


\section{Conclusion}

Management of chronic osteomyelitis is challenging because relatively avascular bone tissue makes the treatment difficult. Prolonged appropriate antibiotics based upon culture and sensitivity in addition to appropriate surgical methods (debridement, pus drainage and skin or bone grafting) is associated with better cure rates. Inadequate treatment is associated with complications like remissions. Chronic pain and loss of functionality may be seen in some patients and in severe cases radical surgeries like amputations may be required.

\section{Conflict of Interest: None}

\section{References}

1. Harik NS, Smeltzer MS. Management of acute hematogenous osteomyelitis in children. Expert review of anti-infective therapy. 2010;8(2):175-181.

2. Khan K, Wozniak SE, Mehrabi E, Giannone AL, Dave M. Sternoclavicular Osteomyelitis in an Immunosuppressed Patient: A Case Report and Review of the Literature. The American Journal of Case Reports. 2015;16:908-911.

3. Annen MJ, Johnston MJ, Gormley JP, Silverman E. Acute Hematogenous Osteomyelitis Presenting as a "Cold" Rib in a Child. World Journal of Nuclear Medicine. 2017;16(2):160-162.

4. Calhoun JH, Manring MM, Shirtliff M. Osteomyelitis of the Long Bones. Seminars in Plastic Surgery. 2009;23(2):59-72.

5. Sendi P, Friedl A, Graber P, Zimmerli W. Reactivation of dormantmicroorganisms following a trauma. Pneumonia, sternal abscess and calcaneus osteomyelitis due to Mycobacterium tuberculosis. Neth J Med. 2008Sep;66(8):363-4.

6. Olson ME, Horswill AR. Staphylococcus aureus Osteomyelitis: Bad to the
Bone. Cell host \& microbe. 2013;13 (6):629-631.

7. Chiappini E, Mastrangelo G, Lazzeri S. A Case of Acute Osteomyelitis: An Update on Diagnosis and Treatment. NielsenSaines K, ed. International Journal of Environmental Research and Public Health. 2016;13(6):539.

8. Casqueiro J, Casqueiro J, Alves C. Infections in patients with diabetes mellitus: A review of pathogenesis. Indian Journal of Endocrinology and Metabolism. 2012;16(Supp11):S27-S36.

9. Lee YJ, Sadigh S, Mankad K, Kapse N, Rajeswaran G. The imaging of osteomyelitis. Quantitative Imaging in Medicine and Surgery. 2016;6(2):184-198.

10. Hatzenbuehler J, Pulling TJ. Diagnosis and management of osteomyelitis. Am Fam Physician. 2011 Nov 1;84(9):102733.

11. Walter G, Kemmerer M, Kappler C, Hoffmann R. Treatment Algorithms for Chronic Osteomyelitis. Deutsches Ärzteblatt International. 2012;109(14):257-264.

12. Felman AH, Shulman ST. Staphylococcal osteomyelitis, sepsis, and pulmonary disease. Observations of 10 patients with combined osseous and pulmonary infections. Radiology. 1975 Dec;117(3 Pt 1):649-55.

13. Rao N, Ziran BH, Lipsky BA. Treating osteomyelitis: antibiotics and surgery. Plast Reconstr Surg. 2011 Jan;127 Suppl 1:177S-187S.

14. Huang C-C, Tsai K-T, Weng S-F, et al. Chronic osteomyelitis increases long-term mortality risk in the elderly: a nationwide population-based cohort study. BMC Geriatrics. 2016;16:72.

15. Shen Y, Hu X, Wen C, Ning F, Yu D, Qin F. Treatment of post-traumatic chronic calcaneal osteomyelitis and soft tissue defect by using combined muscle and skin flaps of calf Zhongguo Xiu Fu Chong 
Jian Wai Ke Za Zhi. 2013 Sep;27(9):10614.

16. Lu Y, Cen Y, Qing Y, Wang H. Surgical treatment of chronic osteomyelitis of the skull Zhongguo Xiu Fu Chong Jian Wai Ke Za Zhi. 2011 May;25(5):558-61.

17. Fritz JM, McDonald JR. Osteomyelitis: Approach to Diagnosis and Treatment. The Physician and sportsmedicine. 2008;36(1):nihpa116823.

18. Thanni L. Bacterial osteomyelitis in major sickling haemoglobinopathies: geographic difference in pathogen prevalence. African health sciences. 2006;6(4):236-239.

19. Ikpeme IA, Ngim NE, Ikpeme AA. Diagnosis and treatment of pyogenic bone infections. Afr Health Sci. 2010 Mar;10(1):82-8.

20. Barshes NR, Mindru C, Ashong C, Rodriguez-Barradas M, Trautner BW. Treatment Failure and Leg Amputation Among Patients With Foot Osteomyelitis. Int J Low Extrem Wounds. 2016 Dec;15(4):303-312. 